\title{
A mixed analysis approach to elucidate the multiple chronic condition experience of English- and Spanish-speaking older adults
}

This article was published in the following Dove Medical Press journal: Clinical Interventions in Aging

\author{
Kamilah B Thomas-Purcell ${ }^{1,2}$ \\ Robin J Jacobs ${ }^{3}$ \\ Tyler L Seidman ${ }^{2}$ \\ Amarilis Acevedo ${ }^{2}$ \\ Drenna Waldrop-Valverde ${ }^{4}$ \\ Raymond L Ownby ${ }^{2}$ \\ 'Department of Health Science, \\ College of Health Care Sciences, \\ Nova Southeastern University, Fort \\ Lauderdale, FL, USA; ${ }^{2}$ Department \\ of Psychiatry and Behavioral \\ Medicine, Dr. Kiran C. Patel College \\ of Osteopathic Medicine, Nova \\ Southeastern University, Fort \\ Lauderdale, FL, USA; ${ }^{3}$ Department of \\ Medical Education, Dr. Kiran C. Patel \\ College of Osteopathic Medicine, \\ Nova Southeastern University, Fort \\ Lauderdale, FL, USA; ${ }^{4}$ Nell Hodgson \\ Woodruff School of Nursing, Emory \\ University, Atlanta, GA, USA
}

Background: Having multiple chronic conditions (MCCs) is the most common health condition in older adults. The management of each condition presents many challenges. A key factor in patients' ability to manage their own health care is their level of health literacy.

Purpose: The purpose of this mixed analysis study was to elucidate the experience of older adults living with MCCs and identify associations among the experiences to determine targets for future mobile health (mHealth) interventions focused on MCCs and health literacy.

Participants and methods: Using the Abilities, Skills, and Knowledge Model as a framework, semi-structured interviews were completed with 25 older adults in English and Spanish. Interviews were analyzed using inductive thematic analysis and hierarchical cluster analysis. Results: The main themes from these results included 1) Reflections of chronic disease; 2) Emotional aspects of chronic disease; 3) Physical barriers to well-being; 4) Quality-of-care factors; and 5) Cognitive strategies for self-management. Qualitative results highlighted the importance of a multi-targeted approach to chronic disease self-management (CDSM). Cluster analysis identified associations within the qualitative data, revealing the importance of the subthemes related to coping with pain and the impact of the patient-provider relationship on treatment adherence.

Conclusion: Results support the utility of mHealth interventions to improve health literacy and promote CDSM.

Keywords: multimorbidity, mHealth, self-management, qualitative, cluster analysis, treatment adherence

\section{Introduction}

\section{Multiple chronic conditions}

As the population ages in many developed countries, persons with multiple chronic conditions (MCCs) become a larger proportion of older adults. Having MCCs or multimorbidity, that is, more than one chronic health condition, is one of the most common chronic conditions in older adults. ${ }^{1,2}$ Between 1985 and 2005, the prevalence of chronic diseases doubled and the number of patients with four or more chronic conditions increased by $300 \%$ in the USA. ${ }^{3}$ Older persons with MCCs have to cope with a complex array of medications, dietary and exercise recommendations, relations with health care providers, and insurance regulations in caring for their health. ${ }^{4,5}$ These demands arise in the context of greater requirements for patients to manage their own health care, as hurried providers spend less time providing education and support. ${ }^{1}$ In 2001, the Institute of Medicine (IOM) published a landmark report emphasizing the complex needs of people living with MCCs. ${ }^{3}$ Since the release of the report, there has been little progress in the development of effective care models for this population.
Correspondence: Kamilah B

Thomas-Purcell

CCR 430, Nova Southeastern University, 330 I College Avenue, Fort Lauderdale,

FL 33314, USA

Tel +I 9542621735

Fax + I 9542623257

Email kthomaspurcell@nova.edu 
Research suggests that practicing clinicians are uncertain about utilizing disease-specific guidelines with their older patients with multiple conditions. ${ }^{6}$ The need to help patients manage MCCs is clear, but precisely how to address this need requires further investigation. More empirical evidence, clear guidelines, and effective approaches should be available to improve quality of care. ${ }^{6}$

By itself, providing the highest quality of care will not guarantee improved health outcomes for people with MCCs. Positive outcomes require patients to be active participants in their care. Self-management relates to "the tasks that individuals must undertake to live well with one or more chronic conditions". ${ }^{7}$ While specific health conditions may require specific management skills (eg, checking one's blood sugar in diabetes), common issues such as fatigue, depression, stress, sleep disturbance, and treatment adherence cut across illnesses and can be addressed in a single intervention. Several chronic disease self-management (CDSM) interventions have been developed and tested. ${ }^{8-11}$ However, the reach of these interventions is limited due to the in-person group format that makes it less accessible for persons with limited time or transportation.

\section{Mobile health}

There is a need to improve the delivery of health and social services for older adults with MCCs. Mobile health (mHealth) is a medical and public health practice supported by mobile devices such as mobile phones, patient-monitoring devices, personal digital assistants, and other wireless devices. ${ }^{12}$ mHealth has the potential to increase access to CDSM interventions since its applications can provide information and build skills without the limitations of time of day or physical location. ${ }^{13}$ Additional benefits include reaching people in resource-poor settings, delivering interventions to large numbers of people, and claiming people's attention when it is most relevant. ${ }^{14}$

mHealth studies within vulnerable populations have shown promise to engage patients in self-management. A systematic review of diabetes self-management via cell phones found that providing personalized advice and support delivered through a cell phone can help avoid diabetes symptoms by providing timely treatment adjustments and can lead to improved health outcomes. ${ }^{15}$ Despite this evidence, relatively few studies of mHealth interventions have included vulnerable populations or specifically tailored programmatic aspects to fit the cultural, linguistic, literacy/numeracy, or other needs of marginalized groups. ${ }^{16}$

Additional research demonstrated that a computerdelivered tailored information intervention targeting health literacy could improve disease-related conceptual knowledge and medication adherence. ${ }^{17,18}$ Designed to be deployed either as an information kiosk in a clinical office or on the Internet, there is research to support that it could be cost-effective in improving patients' health literacy and adherence. ${ }^{19}$ It is not clear if the interactive, computer-delivered, multimedia approach is effective in improving CDSM skills in persons with low baseline levels of health literacy who especially need CDSM information and skills.

\section{Health literacy}

A key factor in patients' ability to manage their own health care is their level of health literacy. ${ }^{20}$ Health literacy refers to the individuals' ability to acquire, understand, and use health information to make choices about their health care. ${ }^{21}$ It has been related to patients' health status in a variety of conditions with lower levels of health literacy related to poorer health status and outcomes. ${ }^{20}$

Health literacy is thus a key factor in older persons' ability to care for themselves, but troubling evidence suggests many do not have the level of health literacy required to navigate the health care system. The 2003 National Assessment of Adult Literacy in the USA, for example, showed that as many as 75 million Americans lacked health literacy that would allow them to cope with even moderately complex health care problems. ${ }^{22}$ Individuals belonging to racial and ethnic minority groups and older persons were shown, on average, to have even lower levels of health literacy. Substantial numbers of Blacks, Hispanics, and persons 65 years of age or older have levels below basic health literacy.22 This finding implies that these individuals will struggle to use health information for basic self-care tasks such as following directions on how to take a medication. Substantial research and opinion link one's level of health literacy to health disparities. ${ }^{23-27}$ The IOM found that disparities in access to health care, public health, and other services may present implications for the population of persons with MCCs. Therefore, it is important to understand the experience of diverse groups in order to develop culturally appropriate interventions. ${ }^{3}$

The causes of racial and ethnic disparities in health are complex and are, in large part, related to social and economic influences. ${ }^{28,29} \mathrm{~A}$ need remains for an effective way to address disparities in everyday clinical practice. Given the relationship of health literacy to race and ethnicity and their relationship to health status and outcomes, a possible strategy to address disparities may be to improve the individuals' health literacy. Several interventions have been effective in improving health literacy. ${ }^{30,31}$ These studies have employed 
diverse intervention elements ranging from changes in mode of information delivery (eg, including icons or pictographs to illustrate information) to providing information in video format. However, it has been found that intervention studies are rarely informed by a clear definition or model of health literacy, making the relationship of the distinct intervention elements to the overall concept unclear.

\section{Aim}

A key preliminary step in the development of a CDSM mHealth intervention is to understand the specific skills and knowledge that patients need to manage MCCs. This can be achieved by gaining a sound understanding of the experiences and challenges of living with MCCs from the perspective of ethnically diverse older adults.

Several studies in the area of patient perceptions of MCCs focus on clinical outcomes and evaluate effectiveness of specific interventions from the perspective of providers and are quantitative in nature. ${ }^{11}$ Qualitative studies on patient perspectives report on the impact of pain and decreased energy in completing daily activities including tasks required to appropriately and successfully self-manage. ${ }^{11}$ Additional studies examine specific disease topics such as symptom burden, coping, health care practices, care planning, and the care processes desired by older adults. ${ }^{32}$ Overall, the MCC population is characterized by tremendous clinical heterogeneity; therefore, developing a means for determining homogeneous subgroups is an important step in an effort to improve health status. ${ }^{3}$ One step in this direction would be to examine racial and ethnic disparities in persons with MCCs. Relatively few studies have focused on the perspective of ethnically diverse patients with MCCs with research in this area tending to focus on chronic conditions in general. ${ }^{33-35}$ We found no studies that aimed to identify associations within the patient perspective data using cluster analysis. This study intends to help fill this gap. The purpose of this mixed methods study was to elucidate the experience of ethnically diverse older adults living with MCCs and identify associations among the experiences in order to determine targets for a tailored mHealth intervention focused on MCCs and health literacy.

\section{Participants and methods Participants}

Study recruitment methods consisted of contacting individuals from a previous study during which they had completed a survey on health conditions and symptoms as well as measures of health literacy. Purposive sampling ensured that individuals with diverse health conditions provided a wide range of experiences with chronic conditions. Targeted recruitment guaranteed diversity with respect to age, gender, race, and ethnicity. Patients signed written informed consent forms after discussion with a member of the research team. Each participant received a copy of the study information for future reference.

All interviews were conducted by $\mathrm{PhD}$-level members of the research team and took place in offices located on a university campus in south Florida. Participants received $\$ 40$ cash to compensate them for time and transportation.

Twenty-five older adults with MCCs participated in semistructured interviews in English $(\mathrm{n}=15)$ and Spanish $(\mathrm{n}=10)$ between 2017 and 2018. "Older adults" were defined as those 50 and older for this study in order to find a broad range of individuals who could provide the most generalizable results. To be eligible to participate, individuals had to meet the following criteria: 1) have at least two chronic diseases, 2) be at least 50 years old, 3) English or Spanish speaking, and 4) able to provide informed consent. Patients with any psychiatric or cognitive disorder impairing cognition or the inability to provide informed consent were excluded.

\section{Procedures}

All study procedures followed a protocol approved by the Institutional Review Board of Nova Southeastern University. The study employed an exploratory cross-sectional study design incorporating the qualitative approach of semi-structured interviews followed by a cluster analysis to support the identification of associations within the qualitative data. The qualitative approach allowed for a richer and in-depth exploration of the CDSM experience of older adults. Previous literature suggests that 5-25 participants are adequate for this type of qualitative scientific inquiry. ${ }^{36-38}$ In this study, interviews were continued until we found that we had thoroughly explored all emergent themes raised by the participants.

\section{Conceptual framework and instrument}

The Abilities, Skills, and Knowledge (ASK) model can inform the development of health literacy interventions. The model states that health literacy is a specific form of expertise ("expert skill or knowledge; [...] know-how") that gives patients the capacity to obtain and use health information in ways that ensure their desired health outcomes. ${ }^{24-27}$ The ASK model explicitly defines this form of expertise as depending on general cognitive abilities (eg, attention, memory, verbal and quantitative reasoning), various skills (eg, reading, arithmetic, information search), and conceptual 
knowledge (eg, facts about health conditions, treatments, or health promotion). This model thus combines abilities, skills, and knowledge in defining the complex activities involved in obtaining and using health information to maintain or improve one's health.

The semi-structured interview guide used the ASK model as a framework and was based on existing literature in the field of CDSM. ${ }^{11,39-41}$ The guide ensured review of relevant problems and needed skills or knowledge while providing the flexibility to explore participants' individual experiences. Examples of coping abilities questions included, "What was your reaction to hearing that you have a chronic disease?" and "What sorts of emotional or psychological symptoms do you have or have you experienced?"; examples of skills questions included, "What are some of the things you do to manage your symptoms?" and "How easy or difficult is it for you to manage the side effects of your medications?"; and examples of knowledge questions included, "What tests did the doctors perform to reach a diagnosis?" and "How well do you understand how each of your illnesses works in your body?".

\section{Analyses}

The English interview guide was translated to Spanish by a native Spanish-speaking member of the research team. Interviews were conducted in the participant's preferred language. They lasted $\sim 1$ hour and were audio recorded and transcribed verbatim by a transcription company. Spanish transcripts were then translated to English by the same transcription company. Content was independently read and coded by two members of the research team to uncover and evaluate emergent themes. Reading each narrative line by line, each reader independently selected phrases relevant to the study aim. The two member coding team then met via telephone conference to discuss the selected phrases and extracted meaning units, condensing the content-rich text into a code that highlighted the core meaning of the text. ${ }^{42}$ The codes were reviewed for consistency and refined. Any discrepancies were resolved by consensus. The final codes were entered into NVivo qualitative data management software for organization and management and were further refined. ${ }^{43}$ Finally, using an iterative process the codes were organized into themes.

Numerous codes related to specific chronic conditions were elicited, including hypertension, high cholesterol, or other cardiovascular disease, arthritis, diabetes, and lung diseases. The frequency with which codes occurred together in interviews was calculated. Using NVivo, the number of times that participants discussed arthritis and pain in the same coding segment was tallied. A matrix of these co-occurrences was then exported for further analysis using SPSS 24.0. ${ }^{44}$ Given the complexity and number of coded segments, hierarchical clustering was used to evaluate how codes were linked at various frequencies and to assess how broader themes might be related to health conditions and cross-cutting problems. ${ }^{45}$ In this case, cluster analysis served as an exploratory tool to support the identification of associations within qualitative data.

Hierarchical clustering is a technique that allows the creation of groupings of entities based on their "distances" from each other. In these analyses, distance was defined as how frequently codes occurred together, so that two codes that were frequently mentioned together would be considered close, while those that were infrequently mentioned together were farther away. We used a distance measure suitable for count data, phi squared, as our data consisted of counts of the number of times themes occurred together. ${ }^{46}$

The cluster solution was calculated by first assuming that each code was its own distinct cluster. The two codes that were closest were then placed together into a cluster at a higher level in the hierarchy (Figure 1). At the next step, either another code that was very close to the first two was added to the cluster, or two different themes that were close to each other but distant from others were placed into a new cluster. This process continued until all cases were linked. A graphical representation (dendogram) of how the clustering proceeded facilitates understanding of how themes are linked (Figure 1). We explored several clustering strategies and judged that complete linkage clustering produced a solution that was most readily interpretable and that clearly reflected our participants' experience.

\section{Results}

We elicited numerous codes related to specific chronic conditions, including hypertension, high cholesterol, or other cardiovascular disease, arthritis, diabetes, and lung diseases. The five main themes for this study include 1) Reflections of chronic disease, 2) Emotional aspects of chronic disease (ie, anger, anxiety, depression), 3) Physical barriers to well-being (ie, pain, fatigue, lack of sleep), 4) Quality-ofcare factors (ie, physician-patient relationship, treatment adherence, complexity of care, the need for more information), and 5) Strategies for self-management (ie, positive reappraisal, self-talk, behavioral activation, faith and spirituality). Table 1 presents the demographic characteristics of the patients in this study. There were 6 men (4 English 


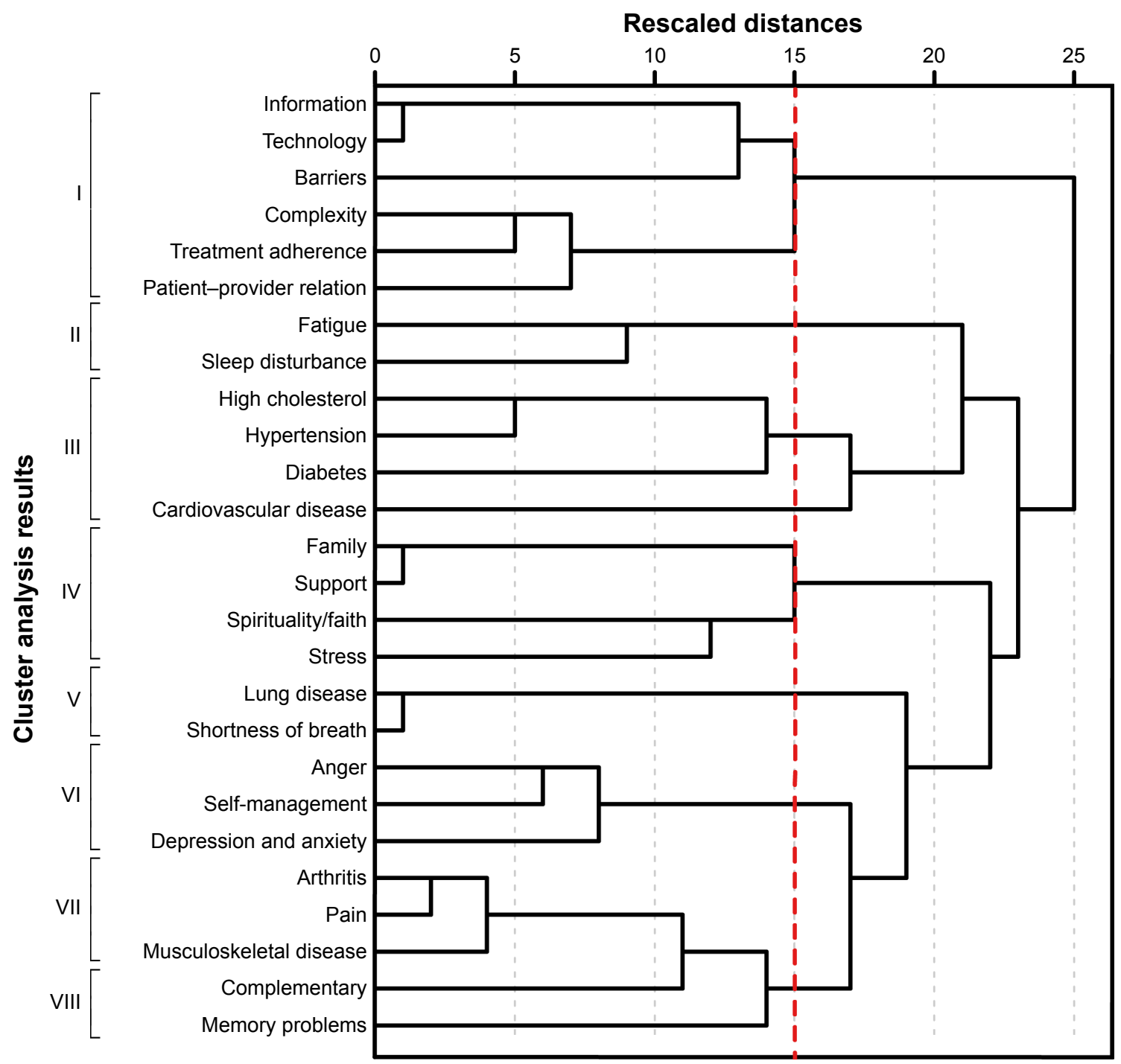

Figure I Hierarchical clustering of older adults' experiences with MCCs.

Note: Roman numerals represent a cluster of information; they were used in the figure to distinguish the groups of information from the distances, which are presented as Arabic numerals.

Abbreviation: MCCs, multiple chronic conditions.

speaking, 2 Spanish speaking) and 19 women (11 English speaking, 8 Spanish speaking). The five African American participants were female. The mean age of the participants was 72 years old ( 75 for English speaking, 68 for Spanish speaking) with a range of $62-89$ years. The average years of education was 12 years with a range of 7-19 years. The number of chronic illnesses reported ranged from one to five with an average of two. Chronic illnesses reported for all participants included diabetes, hypertension, cardiovascular disease, hyperlipidemia, asthma, arthritis, anxiety, and chronic migraines.

\section{Reflections of chronic disease}

Patients reflected on their experiences with chronic illness in many ways. These included mentioning them in the context of family history, prescribed medications, or merely as a laundry list of the ailments they tried to manage, often with suboptimal success. Hypertension was one condition that transcended throughout all of the patient accounts as it was mentioned by the majority of participants:

002: Yeah, and I've been having high blood pressure ever since I was about 14. It runs in my family. [African American female, age 83 years]

019: One of those things which is one of the biggest things you can get, that can - my mom died of high blood pressure. [Spanish speaking, White female, age 65 years]

While sometimes a disease such as hypertension was reported as genetic and early onset, for others it came on later as a result of another illness. 
Table I Demographic characteristics of participants by language spoken

\begin{tabular}{|c|c|c|c|c|}
\hline $\mathbf{N}=\mathbf{2 5}$ & English & Spanish & Total & Percent \\
\hline \multicolumn{5}{|l|}{ Age } \\
\hline $50-60$ & I & I & 2 & 8 \\
\hline $61-70$ & 2 & 6 & 8 & 32 \\
\hline $7 \mid-80$ & 6 & 3 & 9 & 36 \\
\hline $81-90$ & 6 & 0 & 6 & 24 \\
\hline \multicolumn{5}{|l|}{ Sex } \\
\hline Female & 11 & 8 & 19 & 76 \\
\hline Male & 4 & 2 & 6 & 24 \\
\hline \multicolumn{5}{|l|}{ Race } \\
\hline African American & 5 & 0 & 5 & 2 \\
\hline White & 10 & 10 & 20 & 80 \\
\hline \multicolumn{5}{|l|}{ Education } \\
\hline Less than high school & 2 & 5 & 7 & 28 \\
\hline High school/GED & 8 & 3 & 11 & 44 \\
\hline Some college & 2 & I & 3 & 12 \\
\hline College/graduate school & 3 & I & 4 & 16 \\
\hline
\end{tabular}

Abbreviation: GED, General Educational Development.

020: And after the last surgery I had done, which was the prostate one, I began to have problems with high blood pressure. [Spanish speaking, White male, age 68 years]

For some, it was the opposite. Their first chronic illness was hypertension, and other conditions then followed.

023: I started with high blood pressure when I was approximately 48 years old, and sugar was after 3 or 4 years, approximately when I was 52. [Spanish speaking, White female, age 69 years]

Sometimes, the illnesses were brought up in the context of medications:

005: Well, I know the metformin, that's for the diabetes. I take two pills for the high blood pressure, and that's the captopril and atenolol. Those are the only medications that I take is those three. [African American female, age 83 years]

Arthritis was mentioned by several participants:

014: I got osteoarthritis, high blood pressure, slightly high cholesterol [...]. Well, with the arthritis, with the fingers and the shoulders and the knees [...] Both knees are shot. Both shoulders. I've lost the rotary motion in both shoulders. [White male, age 80 years]

022: What I do know is that this disease I have doesn't have a cure. I can just sustain myself so that - that's what I think. So I think that up until now, they haven't found something that will cure rheumatoid arthritis [...]

[White male, age 66 years]

\section{Emotional aspects of chronic disease}

Emotional aspects of coping were prominent in several interviews. In this population, most participants mentioned depression and anxiety.

While some patients mentioned feeling sad, many used the term depression directly. Additional emotional sequelae included anxiety, stress, and anger. In several cases, patients mentioned that these emotions were a result of pain while others stated that these feelings interfered with their sleep patterns.

002: Well I keep kids, and I read, I clean up, I keep this mind occupied. And everything that I think makes me unhappy, I put it on the back burner. I don't want to feel like that. I don't want to feel sad, depressed, and I do get like that. And when I'm depressed, all I do is sleep. Sleep, sleep, sleep, that's all I do is sleep, and I don't want to be bothered. [African American female, age 62 years]

021: Sometimes when the pain is too strong I want to cry and like I get depressed, like you get depressed but that's for a moment. I encourage myself and that will go away. [Spanish speaking, White female, age 77 years]

022: It's affecting me a lot, yes. Because there are days in which I don't want to - I'm in an state in which - because of the pain in my fingers, my body, as if I've been beaten, and I don't even want to go to work. I go because I have to. And that affects me psychologically, I get depressed and disappointed, and I think, "God, why?" [Spanish speaking, White female, age 66 years]

025: And I also have depression and sometimes I can't sleep at night. [Spanish speaking, White female, age 72 years]

\section{Physical barriers to well-being}

Fatigue, stemming from a variety of chronic illnesses (eg, respiratory conditions) or lack of sleep due to pain, was brought up in seven of the interviews.

020: When I do any little thing - even to mow the lawn, which goes on its own, and it goes with the little strength I use, I feel - I feel tired. [Spanish speaking, White male, age 68 years]

008: Yeah, well, I mentioned to you about having to go - get up at night and sometimes I have trouble falling asleep again and on those days, I feel a little sluggish and tired. [White male, age 68 years] 
Though there were other comments that focused on general problems that cut across health, pain was consistent throughout most of the interviews:

013: Well, like now, the weather's so cold, I have - most of my pains - like this way. I was hurting most of the night, last night, right? And, you know - (In your wrist area?) Yes. Wrist area and everything. [African American female, age 83 years]

027: Pain, a lot of pain all over my body. I sit down and I take too long to stand up. So then I have to do it little by little. My legs hurt, my hips hurt, and all of that hurts [...] It affects me because sometimes I want to do things and I do them and I have to do them little by little. It is very upsetting. [Spanish speaking, White female, age 69 years]

012: I can't say I'm managing it because there are certain medications that I cannot take because of my heart [...] actually the only thing I can take for pain is Tylenol, and it doesn't help. [African American female, age 81 years]

022: When I get a strong pain, what I do is - the doctor told me to take - because that's what helps me, Prednisone - when the pain would be too much, to take one and a half. So what I do is with that, I - it seems the inflammation reduces and the pain improves. But I can't be on medication all my life. Do you understand? [Spanish speaking, White female, age 66 years]

Pain also interfered with sleep:

022: Yes, I can't always sleep well. Sometimes I fall asleep at 3:00 a.m. or 4:00 a.m., and then I only sleep two or three hours, and I get up because I am uncomfortable in bed and everything, and my body aches. Sometimes I am not able to get off the bed because of the pain. [Spanish speaking, White female, age 66 years]

\section{Quality-of-care factors}

Quality-of-care factors are those issues that arise from the need to manage diseases and related problems, such as the need for information, complexity of care, the patientprovider relationship, and self-management. The quality and type of communication with providers varied among the participants. It was an important factor among this sample as it was mentioned in the majority of interviews. Some reported a positive relationship, which allowed them to trust their provider's recommendation for treatment:

013: [...] since I had the diabetes for a while now, I'm pretty much up on - pretty much - the up and downs about it. And, with the heart, I'm watching that too, with my heart doctor. I have a very good heart doctor. And he keeps me up to date on everything. Like next month I go to him again. [African American female, age 83 years]

A few had indifferent attitudes toward their health care providers, while others expressed more negative or distrustful feelings:

010: He came across as I want to cure you. He didn't say those words, but that - the other one was like, "Well, I don't know, maybe, maybe." I don't wanna hear that. I wanna hear that you're gonna work on helping me live, not helping me die. [White female, age 89 years]

014: I think the health providers in this day and age are so damn locked up in trying to earn a living, just the way I used to be, that they can't take the time out with individuals that they used to 40, 50 years ago. [White male, age 80 years]

018: But what I see out there in caregivers, I would rather depend on myself, frankly. You look at these people, and you see the dollar sign on them, and they're only working for the dollar. They pretend. Some of them know how to, most of them don't. It's very obvious. Don't do me any favors. [White female, age 57 years]

Others felt they should challenge recommendations they do not agree with and seek the advice of other members of the health care team.

015: No If I have any issues, we usually talk to the pharmacist, and she can explain it pretty well for us. Sometimes, I think they give me too many medications, and - I don't know if I should, but if I don't want to - If I stop taking them, I just do. And the doctor said if I feel I don't need them, then okay. [White female, age 75 years]

009: [...] And she wanted me to do the surgery two years ago. And she said, "Why are you waiting." And I didn't wanna do it. So now it's two years later, and I went to another doctor. [White female, age 89 years]

\section{Strategies for self-management}

Coping or self-management strategies included positive reappraisal, self-talk to guide behavior, and behavioral activation, often integrated with religious faith or more broadly spiritual beliefs. Positive reappraisal is a critical part of coping that allows participants to adapt to the stress of living with chronic illness and enables participants to find alternate assessment of what is happening to them. An example of positive reappraisal:

014: Eat healthy; have a positive attitude toward it. Don't let it stress you out because there's still more to life than 
really thinking about your illness. As long as you do what you're supposed to do and do it right, I don't think you should have a problem. [White male, age 80 years]

018: Well, in my cases, I say to myself, for instance, this thing in my back is bothering me now. Look how much worse the rest of the world is. Look how much worse everybody else - I watched a friend of mine die of cancer [...] I don't know. But I think I'm comparatively lucky. [White female, age 57 years]

An example of self-talk guiding behavior:

011: And I came, and if I would get a migraine, I was in bed for the day. I didn't care, she'd make a dinner or whatever, and she didn't ever do anything. My dad did everything. But when she got the Alzheimer's, I had to do it, and I said, "Well, I have to do this. I have to handle this." And I did. I just became real strong and did it, but I never thought of myself as a strong person until then. And now, I feel like I can do anything, even if I have a migraine. I'll do it. I have to do it. [White female, age 65 years]

Examples of behavioral activation:

002: You know, I try not to think about it. I stay busy. I stay very busy [...] I keep kids, and I read, I clean up, I keep this mind occupied. [African American female, age 62 years]

009: I read a lot on the Internet, so I know that it's important to keep busy, and do the things you like to do, and be positive in spite of everything. [White female, age 73 years]

018: There are some mornings that I get up - it's very funny - and I get in the car and I say is today the day that I'm gonna get killed? Is today the day that I'm not gonna make it or something? And then after you drive for a while it goes away. Because you can't - if you let it beat on you, it's gonna defeat you. Yeah, I'm a survivor [...] [White female, age 57 years]

Faith- or spirituality-related coping was mentioned in less than half of the interviews. Although spirituality is not in itself considered cognitive, the operations used by the participants were.

005: I just I just began talking to myself, and I'm a believer in God. I just said I'm gonna put it in his hands. Whatever happens, whatever the diagnosis, if I have to have the mastectomy or whatever has to be done, I'm just gonna go ahead and have it done. [African American female, age 83 years]

006: I learned how to walk all over again, a guy I worked with then, he helped out a lot, but I got - to me it's like an inner spirit thing $[\ldots]$ To give me that power to keep me going. [African American male, age 71 years]

013: Well, like I said, you know, like I tell them: we can't help yourself because these things has already occurred in our bodies. So we just have to be patient and give it to the Lord. [African American female, age 83 years]

Overall, participants used either one or a combination of self-management strategies to cope with their multiple health conditions.

\section{Cluster analysis}

Results of the cluster analysis are presented in Figure 1. As the process of hierarchical clustering continues until all clusters are connected, it is necessary to choose a level at which to interpret theme groupings. The level at which we estimated the links between clusters were most meaningful is indicated by the dashed line in Figure 1.

Groupings are indicated at the left and marked with Roman numerals. Cluster I includes a subgroup related to disease information, using technology, and barriers to obtaining the information. It includes a second subgroup of codes related to complexity of care, treatment adherence, and patient-provider relations. Together, codes seem to represent a broad group of problems encountered by patients with respect to understanding their conditions, obtaining care, and adhering to sometimes complex treatment regimens.

Cluster II includes sleep disturbance and fatigue, emphasizing their close relation. Cluster III includes specific conditions that frequently occur together, including elevated levels of cholesterol, hypertension, diabetes, and other forms of cardiovascular disease. Cluster IV links stress, spirituality, and religious faith with family and support more broadly. Cluster V links lung disease with the problem of shortness of breath.

The items in Cluster VI suggest links between anger, depression and anxiety, and various strategies for selfmanagement. Emotional aspects of coping were prominent in several interviews. The broad theme of self-management (an element of cluster VI) was used to describe participants' accounts of how they managed themselves to achieve health goals. In some cases, these included strategies to remember how to adhere to treatment, but this theme's close linkage to anger and depression and anxiety emphasizes the frequency with which participants mentioned the importance of strategies for coping with emotional issues.

Cluster VII links arthritis, pain, and other musculoskeletal disease. Participants also reported different types of pain and discussed ways it affected their physical and emotional 
well-being. The final cluster, VIII, is linked to cluster VII (arthritis and pain). It includes subthemes on the use of complementary or alternative medicines and memory problems.

\section{Discussion}

The purpose of this paper was to report results of our qualitative study of older individuals with chronic health conditions, further supported by cluster analysis. The aim of the research was to better understand the experience of a diverse group of older adults. Several themes were drawn from 25 individual interviews: 1) Reflections of chronic disease, 2) Emotional aspects of chronic disease (ie, anger, anxiety, depression), 3) Physical barriers to well-being (ie, pain, fatigue, lack of sleep), 4) Quality-of-care factors (ie, doctor-patient relationship, treatment adherence, complexity of care, the need for more information), and 5) Strategies for self-management (ie, positive reappraisal, self-talk, behavioral activation, faith and spirituality). Cluster analysis was used to better understand the relations among themes mentioned in individual interviews, using the frequency with which topics emerged together in interviews to generate a hierarchy. The use of cluster analysis tools in qualitative analysis software such as NVivo remains underreported ${ }^{45}$ This study adds to the dearth of mixed methods and mixed analysis literature because it converts qualitative data for use with a quantitative analysis. Most mixed methods studies use a parallel analysis, where qualitative and quantitative analyses are conducted separately, and findings are not compared or combined until the interpretation stage. ${ }^{47}$

In the present study, cluster analysis provided a quantitative strategy that allowed us to explore the ways health conditions, problems, and other factors in health care are related. Some findings are intuitively apparent, such as the link between arthritis and pain. Other links highlight factors related to treatment adherence, such as the importance of the patient-provider relationship and the complexity of managing multiple health conditions. Research has shown that productive patient-provider relationships are associated with greater patient satisfaction, better treatment adherence, and improved outcomes. ${ }^{48,49}$

Finally, the links among family, support, stress, and spirituality/religious faith support the importance of these factors in older persons' coping with health-related stress. Therefore, the important role that families and other caregivers provide in managing chronic conditions must be recognized and supported, as some individuals with MCCs will be limited in their ability to perform self-management due to cognitive decline and severe illness. ${ }^{3}$ The results provide a glimpse of the many factors experienced by an ethnically diverse group of older adults with MCCs. The cluster analysis made connections within the qualitative data that allow for a better understanding of the specific skills and knowledge that patients need to cope with the aspects of their chronic conditions that create barriers to their quality of life.

Results are in many respects similar to those reported in other studies on the needs of individuals with chronic health conditions, especially with respect to the frequency with which conditions occur together and the importance of problems that cut across specific diseases, such as fatigue, depression, and pain. For example, a qualitative study of midlife individuals aged 36-59 evaluated the illness experience of individuals with multiple chronic illnesses.$^{50}$ Their findings paralleled the current study in several instances, including finding that individuals were challenged by the logistics of managing MCCs. Their participants also mentioned problems related to finding and using health condition-related information, and the use of positive appraisals of their situation as a coping mechanism. By contrast, their study concerns about stigma and changes in identity status emerged that were not found. It is possible that the impact of multiple conditions on the identity of middle-aged adults may be greater than that of the older adults included in our study. Support for this interpretation is found in the results of a study by Clarke and Bennett. ${ }^{51}$ In an exploration of the experience of MCCs in older individuals (aged 73 years and older), they found a greater acceptance of multiple health conditions as part of the aging process. This acceptance, however, was accompanied by frustrations related to loss of autonomy and changes in physical appearance and function.

Though mentioned by less than half of participants, an important finding was the importance of spiritual and religious beliefs as a cognitive coping strategy for healthrelated stresses. This finding is similar to Harris et al who investigated religious coping with stress in older adults. ${ }^{52}$ It also reflects an interest in research on coping that suggests that finding meaning in difficult situations may be a positive strategy for coping more generally with stressors. ${ }^{53}$

An interesting observation, not specifically verbalized by participants during interviews, was their resilience in managing their conditions, often accompanied by chronic and sometimes debilitating physical and emotional pain from, for instance, migraines, arthritis, and other musculoskeletal conditions, depression. It may not be the physical tasks such as mastering injection needles or blood pressure cuffs that are problematic, but challenges associated with emotional issues associated with pain, fatigue, and depression. ${ }^{11}$ As mentioned 
previously, participants discussed various self-management strategies including coping strategies of self-talk guiding behavior, behavioral activation, and positive reappraisal. These strategies and subsequent success may be linked to patient resilience. Researchers have found an association between resilience, quality of life, and health promotional behavior. ${ }^{54}$ More research is needed to ascertain which protective factors for resilience can be promoted in this group.

This study is not without limitations. First, findings from a small sample cannot be replicated or generalized to larger populations of adults with chronic conditions who receive care in institutions (eg, hospitals, long-term care facilities), in the home, or in rural settings. Second, the quality of the research depends heavily on the individual skills of the researcher and is not immune to a type of researcher bias known as confirmation bias (what occurs when a research forms a belief and uses respondents' information to confirm this belief). To avoid this, multiple researchers were involved in analyzing the data. Third, the researchers' presence during interviews can affect the participants' responses, albeit difficult to avoid in qualitative inquiry. ${ }^{55}$ Participants may discuss their issues in a manner they think will please the researcher or will be socially acceptable. Fourth, participant responses are often subjective and open to wide interpretation, particularly cultural, making it challenging to make systematic comparisons. Last, hierarchical clustering is a technique that inherently depends on a number of researcher decisions that can affect the results. These include choice of distance measure and type of clustering algorithm. ${ }^{56}$ The clustering algorithm used in this study was chosen due to the exploratory nature of the study and the desire to evaluate the relations of the symptoms at various levels of similarity. The distance measure used was chosen because studies have shown that it is likely to produce compact clusters that facilitate interpretation. ${ }^{56}$ Use of other methods may produce different results.

\section{Conclusion}

In summary, this study provides needed information on the types of problems encountered by older persons in coping with multiple chronic diseases. In these analyses, we supplemented more typical qualitative analysis techniques with a cluster analysis. The qualitative analysis helped develop our understanding of the relations among the various subthemes that emerged in the individual interviews. The cluster analysis helped us understand the cooccurrence of specific problems and emphasized the importance of some subthemes such as those related to coping with pain and the impact of the patient-provider relation on treatment adherence. Implications of this study include increasing health literacy among older adults with MCCs regarding their treatment and management through the design and delivery of mHealth interventions that address the five themes of: 1) Reflections of chronic disease, 2) Emotional aspects of chronic disease, 3) Physical barriers to well-being, 4) Quality-of-care factors, and 5) Cognitive strategies for self-management. Specifically, it is important to discuss not only the physical but also the emotional side effects that may occur because of living with MCCs that emerged in Cluster VII (anger, depression, and anxiety), making it an important area for intervention. Although most participants did not mention religion and spirituality, it may be beneficial to explore its potential to provide a source of strength to help older adult patients cope with the emotional effects of MCCs. Spirituality has been associated with positive effects on mental health including coping, resiliency, and fostering social networks, and plays an important role in effective improvement of chronic disease outcomes among minority populations. It has been found that spirituality allows individuals with chronic pain to accept their condition and give meaning to their lives despite their pain. ${ }^{57}$ The relationship between spirituality and health provides an important perspective for health interventions.

While self-management is an overarching theme for this sample, it is important to consider the nuances in how each person approached self-management when designing tailored interventions for this group. There is an opportunity to incorporate the aforementioned coping strategies of self-talk, behavioral activation, and positive reappraisal into MCC interventions. Additionally, it is important to note that other than a few patients mentioning healthy eating, most patients did not mention how they could treat their chronic diseases outside of medications. Therefore, interventions that stress the importance of physical activity and healthy eating to supplement the use of pharmaceuticals may be useful within this population.

This study was initiated to guide the development of a tailored mHealth intervention that incorporates health literacy and CDSM particularly for those faced with MCCs. The findings of this study elucidate the influence of certain social, cultural, and health literacy factors in understanding and participating in chronic illness self-management. For older adults, mHealth can intervene to improve health literacy and encourage resilience. Results indicate that mHealth will likely be most effective by targeting potential deficits in knowledge acquisition and health literacy as well as address motivators such as social support, coping, self-management strategies, and spirituality. 
The cluster analysis helped us revise the proposed topics for the health literacy intervention. Thus, the clustering served as a useful tool for making sense of a large amount of qualitative interview data. This information has the potential to inform other interventions that seek to improve patient health literacy. Future research should determine if these results are consistent among various ethnic groups or if there are certain themes that are particularly relevant to the ethnic groups who participated in this study. Additional research in this area should focus on addressing health disparities and making clear links between CDSM intervention elements and health literacy.

\section{Acknowledgments}

This study was supported by grants from the US National Heart, Lung, and Blood Institute (R56HL096578 05A1) and the National Institute on Minority Health and Health Disparities (RO1MD010368). The funders had no role in the design of the study, in the collection, analyses, or interpretation of data, in the writing of the manuscript, and in the decision to publish the results.

\section{Disclosure}

Dr Raymond L Ownby reports grants from the US National Institute on Minority Health and Health Disparities, during the conduct of the study, and another from Enalan Communications, Inc., outside the submitted work. In addition, Dr Ownby has a provisional patent application pending. The other authors report no conflicts of interest in this work.

\section{References}

1. Tinetti ME, Fried TR, Boyd CM. Designing health care for the most common chronic condition - multimorbidity. JAMA. 2012;307(23): 2493-2494.

2. Wolff JL, Starfield B, Anderson G. Prevalence, expenditures, and complications of multiple chronic conditions in the elderly. Arch Intern Med. 2002;162(20):2269-2276.

3. US Department of Health \& Human Services. Multiple Chronic Conditions - A Strategic Framework: Optimum Health and Quality of Life for Individuals with Multiple Chronic Conditions. Washington, DC; 2010.

4. Beebe CA, Schmitt S. Engaging patients in education for selfmanagement in an accountable care environment. Clinical Diabetes. 2011;29(3):123-126.

5. Woolf SH, Chan EC, Harris R, et al. Promoting informed choice: transforming health care to dispense knowledge for decision making. Ann Intern Med. 2005;143(4):293-300.

6. Fried TR, Tinetti ME, Iannone L. Primary care clinicians' experiences with treatment decision making for older persons with multiple conditions. Arch Intern Med. 2011;171(1):75-80.

7. Adams K, Grenier AC, Corrigan JM. The First Annual Crossing the Quality Chasm Summit: A Focus on Communities. Washington, DC: National Academies Press; 2004.
8. Lorig KR, Sobel DS, Ritter PL, Laurent D, Hobbs M. Effect of a selfmanagement program on patients with chronic disease. Eff Clin Pract. 2001;4(6):256-262.

9. Lorig KR, Sobel DS, Stewart AL, et al. Evidence suggesting that a chronic disease self-management program can improve health status while reducing hospitalization: a randomized trial. Med Care. 1999; 37(1):5-14.

10. Ory MG, Ahn S, Jiang L, et al. Successes of a national study of the chronic disease self-management program: meeting the triple aim of health care reform. Med Care. 2013;51(11):992-998.

11. Liddy C, Blazkho V, Mill K. Challenges of self-management when living with multiple chronic conditions: systematic review of the qualitative literature. Can Fam Physician. 2014;60(12):1123-1133.

12. World Health Organization. mHealth: New Horizons for Health Through Mobile Technologies: Second Global Survey on eHealth. Geneva; 2011.

13. Jerant A, Sohler N, Fiscella K, Franks B, Franks P. Tailored interactive multimedia computer programs to reduce health disparities: opportunities and challenges. Patient Educ Couns. 2011;85(2):323-330.

14. Wilhide Iii CC, Peeples MM, Anthony Kouyaté RC. Evidence-based mHealth chronic disease mobile app intervention design: development of a framework. JMIR Res Protoc. 2016;5(1):e25.

15. Krishna S, Boren SA. Diabetes self-management care via cell phone: a systematic review. J Diabetes Sci Technol. 2008;2(3):509-517.

16. Peek ME. Can $m$ Health interventions reduce health disparities among vulnerable populations? Divers Equal Health Care. 2017;14(2): $44-45$.

17. Ownby RL, Jacobs RJ, Waldrop-Valverde D, Davenport R, Caballero J. A computer-delivered IMB-based health literacy intervention reduces racial disparities in HIV information. Paper presented at: Annual Adherence Meeting of the International Association of Providers in AIDS Care; June 2-4, 2014; Miami, FL.

18. Ownby RL, Waldrop-Valverde D, Caballero J, Jacobs R. Baseline medication adherence and response to an electronically delivered health literacy intervention targeting adherence. NBHIV. 2012;4:113-121.

19. Ownby RL, Waldrop-Valverde D, Jacobs RJ, Acevedo A, Caballero J. Cost effectiveness of a computer-delivered intervention to improve HIV medication adherence. BMC Med Inform Decis Mak. 2013;13(1):29.

20. Berkman ND, Sheridan SL, Donahue KE, Halpern DJ, Crotty K. Low health literacy and health outcomes: an updated systematic review. Ann Intern Med. 2011;155(2):97-107.

21. U.S. Department of Health and Human Services. Healthy People 2010. 2nd ed. Washington: US Government Printing Office; 2000.

22. Kutner M, Greenberg E, Jin Y, Paulsen C. The Health Literacy of America's Adults: Results from the 2003 National Assessment of Adult Literacy (NCES 2006-483). Washington, DC: US Department of Education, National Center for Educational Statistics; 2006.

23. Pignone M, DeWalt DA, Sheridan S, Berkman N, Lohr KN. Interventions to improve health outcomes for patients with low literacy. A systematic review. J Gen Intern Med. 2005;20(2):185-192.

24. Osborn CY, Cavanaugh K, Wallston KA, et al. Health literacy explains racial disparities in diabetes medication adherence. J Health Commun. 2011;16(Suppl 3):268-278.

25. Osborn CY, Paasche-Orlow MK, Bailey SC, Wolf MS. The mechanisms linking health literacy to behavior and health status. Am J Health Behav. 2011;35(1):118-128.

26. Osborn CY, Cavanaugh K, Wallston KA, White RO, Rothman RL. Diabetes numeracy: an overlooked factor in understanding racial disparities in glycemic control. Diabetes Care. 2009;32(9):1614-1619.

27. Waldrop-Valverde D, Osborn CY, Rodriguez A, Rothman RL, Kumar M, Jones DL. Numeracy skills explain racial differences in HIV medication management. AIDS Behav. 2010;14(4):799-806.

28. Smedley BD, Stith AY, Nelson AR. Unequal Treatment: Confronting Racial and Ethnic Disparities in Health Care. Washington, DC: National Academies Press; 2003.

29. Diez Roux AV. Conceptual approaches to the study of health disparities. Annu Rev Public Health. 2012;33(1):41-58. 
30. Berkman ND, Sheridan SL, Donahue KE, et al. Health literacy interventions and outcomes: an updated systematic review. Evidence report/ technology assessment no. 199. Rockville, MD: Agency for Healthcare Research and Quality; 2011.

31. Sheridan SL, Halpern DJ, Viera AJ, Berkman ND, Donahue KE, Crotty K. Interventions for individuals with low health literacy: a systematic review. J Health Commun. 2011;16(Suppl 3):30-54.

32. Ploeg J, Matthew-Maich N, Fraser K, et al. Managing multiple chronic conditions in the community: a Canadian qualitative study of the experiences of older adults, family caregivers and healthcare providers. BMC Geriatrics. 2017;17(1):1-15.

33. Jacobs RJ, Ownby RL, Acevedo A, Waldrop-Valverde D. A qualitative study examining health literacy and chronic illness self-management in Hispanic and non-Hispanic older adults. J Multidiscip Healthc. 2017;10: 167-177.

34. Phillips KL, Rogers CR, Aiken-Morgan AT, Cr R, Rogers CR, Aiken AT. Perceptions of chronic disease among older African Americans: a qualitative analysis. J Health Dispar Res Pract. 2017;10(4):52-60.

35. Loeb SJ. African American older adults coping with chronic health conditions. J Transcult Nurs. 2006;17(2):139-147.

36. Flick U. An Introduction to Qualitative Research. Thousand Oaks, CA: SAGE Publications; 2009

37. Mason M. Sample size and saturation in $\mathrm{PhD}$ studies using qualitative interviews. Forum Qual Soc Res. 2010;11(3).

38. Guest G, Bunce A, Johnson L. How many interviews are enough? An experiment with data saturation and variability. Field Methods. 2006;18(1):59-82.

39. Bayliss EA, Ellis JL, Steiner JF. Barriers to self-management and quality-of-life outcomes in seniors with multimorbidities. Ann Fam Med. 2007;5(5):395-402.

40. Beverly EA, Wray LA, Chiu CJ, Weinger K. Perceived challenges and priorities in co-morbidity management of older patients with type 2 diabetes. Diabet Med. 2011;28(7):781-784.

41. Townsend A, Hunt K, Wyke S. Managing multiple morbidity in mid-life: a qualitative study of attitudes to drug use. BMJ. 2003;327(7419):837.

42. Graneheim UH, Lundman B. Qualitative content analysis in nursing research: concepts, procedures and measures to achieve trustworthiness. Nurse Educ Today. 2004;24(2):105-112.

43. NVivo qualitative data analysis Software; QSR International Pty Ltd. Version 10, 2012. Available from: https:/www.qsrinternational.com/ nvivo/what-is-nvivo. Accessed February 12, 2019.
44. IBM Corp. Released 2016. IBM SPSS Statistics for Windows, Version 24.0. Armonk, NY: IBM Corp. Available from: https:/www-01.ibm. com/support/docview.wss?uid=swg21476197. Accessed February 12, 2019.

45. Macia L. Using clustering as a tool: Mixed methods in qualitative data analysis. The Qualitative Report. 2015;20(7):1083-1094. Available from: http://www.nova.edu/ssss/QR/QR1020/1087/macia1083.pdf. Accessed February 12, 2019

46. SAS Institute. SAS/STAT 13.1 User's Guide. Cary, NC: SAS Institute; 2013.

47. Henry D, Dymnicki AB, Mohatt N, Allen J, Kelly JG. Clustering methods with qualitative data: a mixed-methods approach for prevention research with small samples. Prev Sci. 2015;16(7):1007-1016.

48. Brown JB, Stewart M, Ryan BL. Outcomes of Patient-Provider Interaction. Mahwah, NJ: Erlbaum; 2003.

49. Rao JK, Anderson LA, Inui TS, Frankel RM. Communication interventions make a difference in conversations between physicians and patients: a systematic review of the evidence. Med Care. 2007; 45(4):340-349.

50. Markle GL, Attell BK, Treiber LA. Dual, yet Dueling illnesses: multiple chronic illness experience at midlife. Qual Health Res. 2015; 25(9):1271-1282.

51. Clarke LH, Bennett E. 'You learn to live with all the things that are wrong with you': gender and the experience of multiple chronic conditions in later life. Ageing Soc. 2013;33(2):342-360.

52. Harris GM, Allen RS, Dunn L, Parmelee P. "Trouble won't last always": religious coping and meaning in the stress process. Qual Health Res. 2013;23(6):773-781.

53. Folkman S, Moskowitz JT. Coping: pitfalls and promise. Annu Rev Psychol. 2004;55(1):745-774.

54. Cal SF, deSá LR, Glustak ME, Santiago MB. Resilience in chronic diseases: a systematic review. Cogent Psychology. 2015;2(1):1024928.

55. Patton MQ. Qualitative Evaluation and Research Methods. 4th ed. Newbury Park, CA: Sage; 2014.

56. Everitt BS, Landau S, Leese M, Stahl D. Cluster Analysis. 5th ed. New York, NY: Wiley; 2011

57. Sorajjakool S, Aveling L, Thompson KM, Earl A. Chronic pain, meaning, and spirituality: a qualitative study of the healing process in relation to the role of meaning and spirituality. J Pastoral Care Counsel. 2006; 60(4):369-378
Clinical Interventions in Aging

\section{Publish your work in this journal}

Clinical Interventions in Aging is an international, peer-reviewed journal focusing on evidence-based reports on the value or lack thereof of treatments intended to prevent or delay the onset of maladaptive correlates of aging in human beings. This journal is indexed on PubMed Central, MedLine,

\section{Dovepress}

CAS, Scopus and the Elsevier Bibliographic databases. The manuscript management system is completely online and includes a very quick and fair peer-review system, which is all easy to use. Visit http://www.dovepress. com/testimonials.php to read real quotes from published authors. 